\title{
La formación del profesional desde la superación en la universidad de Ecuador
}

\author{
Dr. Felipe Manuel León Cáceres \\ manuel_leonc@hotmail.com \\ Orcid. 0000-0003-4760-9342 \\ Universidad de Guayaquil \\ Dr. Milton Doroteo Cayambe Guachilema \\ Cayambe1970@hotmail.com \\ Orcid.org/0000-0003-4772-894X \\ Universidad de Guayaquil \\ MSc. Douglas Mario Alvarado Herrera \\ domaalhe@gmail.com \\ Orcid. 0000-0001-9203-7975 \\ Universidad de Guayaquil \\ MSc. Manuel Pedro Palacios Noboa \\ peter_pala@msn.com \\ Orcid. 0000-0003-0244-4708 \\ Universidad de Guayaquil \\ MSc. Ronny Cesar Alvarado Pincay \\ ronnyalbapin@gmail.com \\ Orcid. 0000-0002-3958-9778 \\ Universidad de Guayaquil \\ MSc. Jorge Humberto Chasi Zurita \\ jorge.chasiz@ug.edu.ec \\ Orcid. 0000-0002-1050-1605 \\ Universidad de Guayaquil \\ MSc. Angélica del Rocio Tomalá de la Cruz \\ angelicatomala@hotmail.com \\ Orcid.0000-0003-0095-7710 \\ Universidad de Guayaquil
}

\section{RESUMEN}

La superación de los docentes constituye uno de los aspectos esenciales para el adecuado desempeño profesional. De modo que las fisuras tanto teóricas, como pedagógicas o didácticas, tengan respuesta en su auto estudio y reflexión a través de cursos de postgrados y talleres metodológicos. El objetivo del artículo consiste en: desarrollar una superación 
profesional del docente de la educación superior, que permita elevar mejores resultados en la formación de los egresados. La aplicación de instrumentos empíricos y revisión de documentos, posibilitó determinar los problemas de aprendizaje en algunos estudiantes de carreras universitarias, con mayor énfasis, reveladas en las limitaciones en el uso de la lengua materna tanto oral como escrita que afectan la calidad de la asimilación de conocimientos. Por tanto, instrumentar talleres teóricos y metodológicos que den prioridad a las fisuras de los maestros referidas a los fundamentos de la lingüística del texto y la pedagogía, posibilitará a mediano plazo, brindar solución a las debilidades de los estudiantes y al mismo tiempo, revelar niveles superiores de desempeño en los docentes para el logro del desarrollo como categoría pedagógica.

Palabras claves: superación; pedagógica; docentes, estudiantes; desarrollo. 


\title{
The profesional formation since the capacitation in the university in Ecuador
}

\begin{abstract}
The upgrading courses given to professors constitutes one of the essential aspects for their adequate professional performance, so that the theoretical and pedagogical gaps will be solved through their self studies and reflection developed in the postgraduate courses and methodological workshops. The objective of this article is to develop professional upgrading courses to professors from the tertiary education so that better results could be obtained in the formation of university students. The application of empirical instruments and the revision of documents gave way to the determination of the students' learning problems in some university majors, revealed with great emphasis in the limitation of the use of the mother tongue either oral or written which affects the quality of the assimilation of knowledge. Thus the implementation of theoretical and methodological workshops will give solution to the students' weaknesses and at the same time it will reveal higher levels in the professor's performance.
\end{abstract}

Key words. upgrading courses; pedagogy; teachers; students; development.

Artículo recibido: 15 noviembre. 2021 Aceptado para publicación: 10 diciembre 2021 Correspondencia: manuel_leonc@hotmail.com Conflictos de Interés: Ninguna que declarar 


\section{INTRODUCCIÓN}

El proceso de formación del profesional que se desarrolla en la educación superior constituye un espacio de construcción de significados y sentidos entre los sujetos participantes que implica el desarrollo humano progresivo, lo que se puede explicar desde aportes diversos para su solución, en aras de reconocer este proceso como consciente, complejo, holístico y dialéctico.

A través de esta formación, es que los sujetos desarrollan el compromiso social y profesional, la flexibilidad ante la cultura, la trascendencia en su contexto, toda vez que elevan su capacidad para la reflexión divergente y creativa, para la evaluación crítica y autocrítica, para solucionar problemas, tomar decisiones y adaptarse flexiblemente a un mundo cambiante.

Se asume que alcanzar una integralidad en la formación profesional a nivel universitario implica, ante todo, formar un profesional comprometido con su labor y sociedad en que se inserta, flexible y trascendente, independientemente de la especificidad que impone cada profesión y sus contextos.

Pero para ello, resulta necesario que el currículo universitario contenga las cualidades que se aspiran formar en los estudiantes, con lo cual se puede precisar cuál es el tipo de profesional que se quiere y cómo se estructura esta aspiración en todo currículo, permitiendo cumplir su encargo de orientador en la dinámica de la formación de los profesionales sobre la base de dichas cualidades.

Se coincide con el pedagogo Gimeno Sacristán cuando señala que "los debates esenciales en torno a los currículos en la actualidad, como no podía ser de otro modo, están muy estrechamente relacionados con los cambios culturales, políticos, sociales y económicos que están afectando a las sociedades desarrolladas y que tienen como primera consecuencia la revisión del papel asignado a la escolarización y a las relaciones entre esta y los diferentes aspectos que en ella se entrecruzan: profesores, organizaciones, relaciones con la comunidad. etc.". ( S. 2003)

Los cambios curriculares en la educación superior deben ser expresión de la contextualización de las universidades de acuerdo con la complejidad de los escenarios en los que se desarrolla. El entorno económico, político y sociocultural condiciona los procesos educativos en estas instituciones, sin embargo, la universidad no debe asumir 
una posición adaptativa al entorno, sino que debe investigarlo con profundidad para generar los procesos de su transformación.

No es posible olvidar que la formación es a lo largo de toda la vida, no termina nunca y si el ser humano, quiere continuar su camino vital, necesita actualizar sus conocimientos y abrirse a las nuevas realidades que, en cada momento, presiden el desarrollo de la civilización. Se considera también un proceso y resultado que contribuye a preparar al hombre en todos los aspectos de su personalidad, a partir de poder ofrecer instrucción y educación como categorías dialécticas y pedagógicas.

Valiosas han sido las contribuciones en Cuba de (Mariño, G ,1988), (Flórez R., 1990), (Restrepo, M, 1999), (Álvarez de Zayas, 1999), Fuentes G. (2002), (Horruitiner S., 2009) entre otros, enfatizan en el proceso de formación basado en principios científicos, humanísticos y éticos, fundamentados en el respeto a la vida y a la dignidad humana. Chávez brinda una conceptualización interesante sobre formación: "un sistema con un enfoque integral que garantiza el desarrollo multilateral de la personalidad, la elevación de la efectividad de la educación, de la instrucción y su optimización" (Chávez, 2005). En la interpretación se aprecia un nivel de generalidad referido a la formación como proceso institucional y no dirigido específicamente a la formación de los individuos.

Por otra parte, Horruitiner plantea que en una universidad "la formación de profesionales posee como cualidad fundamental la profunda formación básica con dominios de los aspectos esenciales para su ejercicio profesional, asegurando la capacidad de desempeñarse con éxito en las diferentes esferas de su actividad laboral". (Horruitiner, 2009); por lo que se requiere contar con profesores preparados profesionalmente, con una adecuada formación pedagógica que integren la instrucción y la educación en la labor educativa, esto posibilitará una motivación y preparación de los estudiantes.

Por otra parte en Ecuador, desde lo sociológico, la política del Plan del Buen Vivir estipula en el objetivo 3.1 numeral 3 lo siguiente: "Incentivar la implementación de procesos de desarrollo profesional, formación continua, evaluación, certificación y recategorización laboral para los profesionales de la educación y la salud y para los profesionales o técnicos de servicios de atención y cuidado diario" (Plan Nacional del Buen Vivir (2014-2017), se enfatiza en la necesidad de capacitar a los docentes para asumir un desempeño profesional acorde con las exigencias sociales. 
De modo que desde esta ley Art. 156, se reconoce la importancia de la capacitación y perfeccionamiento permanente e incluso, se alude a la formación como categoría pedagógica. Desde esta perspectiva, se utilizan términos similares a los que se utilizan en los estudios en Cuba, y se coincide con la misma intención y objetivo.

Algunos estudios aluden que "la superación es un proceso permanente de adquisición, estructuración y reestructuración de conocimientos, habilidades y valores para el desarrollo y desempeño de la función docente”. (De Lella, 2003). Por ello, se requiere la elaboración de estrategias, metodologías, a través de un componente crítico, tendiendo puentes entre la teoría y la práctica, que sirvan a la teoría para corregir, comprobar y transformar la práctica, en interrelación dialéctica con la comunicación entre los sujetos, como refiere Gorodokin (2005) y se coincide con este criterio.

Desde esta óptica, González Rey F., refiere que la comunicación como proceso y en particular la comunicación desarrolladora, es precisamente aquella en que los sujetos implicados promueven motivos específicos hacia el proceso interactivo, lo que implica la capacidad de entrar en un contexto personalizado de profundo valor motivacional y a través de esta motivación compartida, expresar intereses personales que se integrarán en el vínculo interactivo. Esta idea es la que se pretende demostrar a través de la superación de los docentes.

El papel mediador de los signos y las herramientas del proceso de aprendizaje resulta muy importante para el logro de resultados superiores en la formación de los egresados universitarios. La relación del hombre con los objetos de la cultura está mediatizada con la mediación instrumental (signos y herramientas) y la mediación social (la relación con los otros), de ahí que "la cultura crea formas especiales de comportamiento, modifica las funciones psíquicas, construye nuevos estratos en el sistema de desarrollo del comportamiento del hombre" (Vigotsky, 1998)

Investigadores en el plano internacional y regional como: (Aguerrondo, 2002); (Ávalos, 2007), (Terigi, 2010), Vaillant, (2013) valoran en sus estudios, que se requiere alcanzar un concepto de desarrollo profesional y educación continua, que se ha construido colocando al docente como sujeto en formación, para romper con las visiones tradicionales de la capacitación como medio para compensar deficiencias, o como vía para comunicar los cambios normativos en el currículo y su desarrollo. 
En este sentido, el Proyecto Estratégico Regional sobre docentes UNESCOOREALC/CEPPE (2011) en Argentina, apunta que el desarrollo de los profesionales de la educación a lo largo de la vida, lo cual requiere esfuerzos sostenidos en el marco de políticas docentes articuladas, bien coordinadas y eficientes.

Estas razones, permiten destacar que, desde las instituciones educativas y sus directivos, es imprescindible determinar que la formación continua es significativa, porque incrementa la calidad de la educación en el aula, por lo que se desplaza la perspectiva de lo remedial a favor de una visión que privilegia el impulso de “...aquellas actividades conscientes y planificadas dirigidas intencionalmente al beneficio de individuos, grupos o escuelas..." (Day, 2004).

En la actualidad el docente requiere no solo ser un mero transmisor del conocimiento, sino un facilitador de aprendizaje, colaborador, tutor guía, especialista en contenidos, evaluador del proceso de aprendizaje, inclusive asumir un rol protagónico en su proceso de formación. De ahí la necesidad de elaborar e implementar estrategias pedagógicas o didácticas para revertirlas a partir de la superación.

Los investigadores consideran la formación, como el resultado de un conjunto de actividades organizadas de forma sistemática y coherente, que le permite al individuo poder actuar de manera consciente y creadora; que garantiza la preparación del hombre como un sujeto activo de su aprendizaje y desarrollo, por medio de las relaciones que se establecen entre formación, educación y desarrollo, categorías pedagógicas esenciales de la investigación

Se asume la definición revelada por Antón (2010) acerca de formación continua como:

"La formación continua del profesorado debe centrarse en el desarrollo profesional, en la cualificación y mejora profesional, en favorecer su capacidad de investigación e innovación y de aplicar y aprovechar los conocimientos derivados de su experiencia docente. Como resultado de la adecuación de sus competencias, serán capaces de identificar y adaptar mejor las actuaciones para la aplicación de su nuevo rol en el proceso de enseñanza-aprendizaje."

Por lo tanto, se declara la formación continua como un sistema orgánico y organizado, donde la estructura y todos sus elementos se sincronizan y contribuyen a 
un solo fin, en donde está involucrada la institución, programas, métodos, procesos y conceptos que definen la formación, no solamente la referida al docente, sino aquella que influye en su desarrollo profesional; por eso, el objetivo del artículo es: desarrollar una superación profesional al docente de la educación superior, en aras del logro de mejores resultados en la formación de los egresados. De modo que resulta necesario adentrarse en el siguiente epígrafe.

\section{MATERIALES Y MÉTODOS.}

Se asumen los fundamentos del enfoque cualitativo que posibilita la utilización de métodos como la participación participante de los usuarios, así como la revisión de los documentos esenciales de las carreras seleccionadas como muestra y población, de la Universidad de Guayaquil, Ecuador. Así como métodos empíricos que corroboren las problemáticas.

La revisión de la maya curricular permite corroborar las pocas horas que se imparten de asignaturas básicas como el lenguaje, sin advertir la significación que cobra un mejor uso de la lengua en el aprendizaje, posibilitando así una mejor comunicación oral y escrita de los estudiantes.

A esto se une que no siempre hay docentes capacitados en el dominio de la didáctica de la Lengua Española que permita un mejor tratamiento. Esta didáctica ha transitado de una didáctica del texto y en la actualidad del habla con fundamentos interdisciplinarios y multidisciplinarios de gran valía.

Desde esta perspectiva y seleccionando un total de 25 docentes de las carreras de Informática y Licenciatura en enfermería como muestra aleatoria, se realizan entrevistas a docentes, y especialistas, de modo que se puedan recoger criterios para determinar prioridades y resolver problemáticas de los estudiantes.

En la entrevista los 15 docentes seleccionados revelaron que no siempre advierten que los problemas del aprendizaje de los estudiantes universitarios, en ocasiones, devienen por las fisuras que se revelan en el uso de la lengua materna, lo que afecta la asimilación de saberes y una mejor independencia para realizar tareas, exámenes, discusiones de proyectos, tesis y otros tipos de informes.

Asimismo, son del criterio y se coincide con las respuestas de los especialistas, que la didáctica de la lengua materna requiere un dominio de muchos contenidos vitales para el desarrollo de la competencia comunicativa y la demostración de destrezas, capacidades, 
habilidades y dominios cognitivos en los estudiantes, que no siempre se promueven en el proceso pedagógico, ni se evalúan.

Un total de 10 docentes manifestaron que no poseen el adecuado desempeño profesional, pues no dominan las categorías de la pedagogía y la didáctica, lo que afecta la calidad de las clases y los niveles de ayuda que requieren los estudiantes en el contexto educativo. Incluso destacan que resulta también necesario, poder atender a los estudiantes teniendo en cuenta los fundamentos psicológicos vitales para la calidad del proceso pedagógico. Los estudiantes seleccionados para la aplicación de la encuesta fueron 58, los cuales se encuentran en el 1 y 2 año de su formación. Los resultados generales demostraron que en ocasiones, son tímidos en la participación durante el proceso de enseñanza aprendizaje y en otras actividades, porque no poseen las herramientas indispensables para comunicarse en los diferentes contextos. A esto se une que les resulta compleja la lectura de diversidad de fuentes y textos para preparar sus presentaciones en power point, o de forma escrita $\mathrm{u}$ oral. 5 estudiantes destacaron que poseen problemas ortográficos y de redacción, así como dificultades para argumentar, exponer habilidades esenciales de la comunicación oral.

Los resultados de las pruebas finales en los dos cursos anteriores, revelan bajas calificaciones en el $40 \%$ de los estudiantes, las cuales se concretan en dificultades de dominios cognitivos porque no llegan a explicar, argumentar o demostrar con ejemplos, a partir de sus explicaciones.

Por tanto, se corrobora la necesidad de superar a los docentes con talleres teóricopedagógicos que les permita actualizarse en los contenidos de la didáctica del texto y al mismo tiempo, en las categorías pedagógicas que tanto deben demostrar en los procesos educativos.

\section{RESULTADOS Y DISCUSIÓN.}

Se sometió a discusión cada uno de los Talleres realizados con los docentes como forma de superación profesional y donde se tuvo en cuenta los resultados de los instrumentos empíricos realizados a especialistas, docentes y estudiantes de la muestra y población determinada. El objetivo de esta superación es: implementar una propuesta teóricometodológica que posibilite una adecuada atención a la lengua materna en aras de lograr mayores niveles de independencia en los estudiantes durante el proceso pedagógico. 
Para discutir y reflexionar en los talleres se realizaron técnicas de grupo para lograr la armonía y tranquilidad de los participantes. El primer taller se dedicó a las categorías de la Pedagogía:

Taller 1. Profundiza en la Pedagogía como Ciencia de la Educación.

Se establece un abordaje de las categorías formación, instrucción, educación, y desarrollo, las cuales tienen plena relación con la Didáctica y sus categorías, pues no pueden verse desvinculadas ambas como Ciencias de la educación, sin obviar, el paradigma psicológico que asume la educación en cada país.

Cada docente reveló su preparación metodológica, destacando la búsqueda en textos clásicos y otros más actuales, como los artículos de internet que corroboran la actualidad de estos saberes a través de investigaciones y tesis de maestrías y doctorado por su trascendencia desde la pedagogía o didáctica. Profundizaron en las definiciones y la importancia que tiene para el adecuado desempeño de los docentes mucho más en la educación superior, donde debe revelar preparación pedagógica y didáctica. El segundo taller referido a:

Taller 2. Sabes acerca de las transformaciones en la Didáctica de la lengua materna y sus relaciones interdisciplinarias, transdisciplinarias y multidisciplinarias.

Las reflexiones realizadas a partir del auto estudio de fuentes actuales y consultadas en internet, corroboró el salto cualitativo logrado en la didáctica de la lengua materna denominada en la actualidad didáctica del texto.

Una definición sobre texto que tiene puntos de contactos con otras regiones e investigadores es la de texto: como un texto coherente que se desarrolla con una intención y finalidad comunicativas, en un contexto dado. De ahí las características del texto.

1. En este sentido, el texto ha cobrado importancia a partir de sus propiedades: La coherencia y la cohesión son características fundamentales de todo texto. La primera manifestada a un nivel profundo y caracterizada por la unidad de sentido; la segunda, al modo en que los elementos del texto están relacionados, gramaticalmente hablando.

2. La intencionalidad se refiere a la actitud de quien produce un texto coherente respecto a los objetivos que persigue o a la realidad de un proyecto determinado.

3. La aceptabilidad se refiere al receptor; este recibe un texto claro y coherente elaborado con determinada intención, y producido en una situación específica: si no sucede así, la comunicación resulta afectada. 
4. La informatividad hace referencia al grado de predicción o probabilidad de determinados elementos o informaciones que aparecen en el texto.

5. La situacionalidad se refiere a la importancia de un texto en el interior de una situación comunicativa concreta.

6. La transtextualidad pone en relación al texto con los demás textos con los que establece relaciones de significado. (Permite reconocer al tex to como perteneciente a un tipo determinado: carta, informe, acta...). Existen diferentes formas de presentación de la transtextualidad:

- Intertextualidad: Copresencia: Presencia de un texto dentro de otro (cita textual, referencia a otro texto, frases en otro idioma, etc....)

- Hipertextualidad: Versiones de una misma historia (parodias, caricaturas...)

- Paratextualidad: Un texto antes que otro. (Exergo, prólogo, epílogo...)

Se interroga desde esta pregunta: ¿Creen ustedes que todos los estudiantes cumplen con estas características cuando elaboran un texto oral?. Esto sirvió para profundizar mucho más en la temática por parte de los docentes, quienes deben dominar estos aspectos para evaluar a sus discentes en el proceso pedagógico a través de talleres, seminarios o proyectos investigativos, donde utilizan el texto oral o escrito. El siguiente taller consistió.

Taller 3. Tipología Textual

Una docente refiere que ha sido interesante y muy contextualizado para el trabajo con cualquier asignatura estudiar profundamente la diversidad textual, la cual se utiliza en ocasiones, pero sin darnos cuenta la significación que cobra en las clases de las asignaturas humanísticas principalmente.

Existe variedad de clasificaciones, desde Teun Van Dijk, (1999), Lotman Luri (2003) hasta Dressler, L (2004) y otros. No obstante, se asume a Roméu, Angelina (2008) quien en el libro Comunicación y texto de Ileana Domínguez, aparece un artículo acerca de este tema.

Esta clasificación alude según el código: existen textos orales, escritos e icónicos o simbólicos de gran utilidad cuando de tecnología de alude. La vida cotidiana la realizamos utilizando los primeros, pero en el proceso pedagógico le indicamos a los estudiantes que entreguen proyectos, trabajos independientes, que realicen entrevistas en sus prácticas laborales sin constatar la importancia de estos tipos de textos. 
Por otro lado, según la forma elocutiva los textos pueden ser: dialogados, narrativos, descriptivos, expositivos, argumentativos. Pero existen irregularidades en los estudiantes para lograr la habilidad de argumentar, donde debe ser capaz de convencer ofreciendo razones confiables, convincentes que sean aceptadas. Asimismo, la demostración es la manera lógica entre los argumentos y las tesis a través de los procesos lógicos del pensamiento inducción, deducción y analogía.

La otra clasificación de los textos es según la función: que responden a la función lingüística de la comunicación. Estos pueden ser informativos, expresivos, poéticos, apelativos estos últimos menos utilizados. Según su estilo funcional: textos coloquiales, publicitarios, oficiales, artísticos.

Estos contenidos permiten profundizar en el último taller donde se retoman las modalidades de la comunicación, por ser muy utilizadas en el proceso pedagógico y en el proceso de enseñanza aprendizaje.

Taller 4. Modalidades de la comunicación oral.

Un docente destaca que hay modalidades para dirigirse a un público como son la oratoria, la conferencia, disertación, que son mucho más utilizadas por los docentes. Sin embargo, el estudiante requiere en el proceso instructivo y educativo conversar, exponer, narrar, describir situaciones de aprendizaje e incluso el docente orienta en ocasiones, para el análisis del tema: el debate, la discusión y el panel donde se preparan todos, pero solo algunos exponen los aspectos esenciales y el resto del grupo realiza preguntas o contesta interrogantes.

Todos aludieron que las utilizan en el proceso pedagógico a partir de un contenido muy representativo, pero no siempre los estudiantes alcanzan calidad en la utilización de estas formas. Incluso, por ejemplo la exposición que tanto se promueve en los seminarios y talleres, no siempre adquiere relevancia por el uso del tono de la voz de los jóvenes o su pobre preparación, además en ocasiones, demuestran pobreza léxica, e inadecuado uso de léxico científico de las ciencias e independencia en su discurso. .

Estas modalidades permitieron a los docentes concretar a partir de las formas de organización de la enseñanza en la educación superior, cómo utilizarlas en talleres, clases prácticas a partir de las técnicas de orientación, donde se revelan formas muy útiles para lograr la discusión y el debate: como son: el panel, el seminario o la mesa redonda. Incluso, debe insistirse con los estudiantes en la necesidad de promover la cultura del 
diálogo donde la escucha como habilidad comunicativa, sea esencial cuando los que exponen emiten criterios, definiciones, de modo que se asimilen nuevos contenidos que les permita luego intervenir, discrepar o argumentar.

En el proceso pedagógico y a través de la instrucción y educación como categorías pedagógicas, se concretan temáticas que pueden potenciar la discusión y el debate. Por tanto, se requiere aprender a debatir, indicando con antelación las bibliografías diversas para profundizar en el tema, respetando los criterios de los otros. Se considera el debate un método pedagógico.

En el taller final los participantes explicitaron que esta superación teórico- metodológica acerca de estos temas de la didáctica del texto, abren un universo de saberes para docentes y estudiantes porque potencian una mejor comunicación entre los alumnos del grupo y al mismo tiempo, entre estos y el profesor, lo que redunda en aprendizaje y desarrollo de la lengua materna en los jóvenes mejorando así, la producción textual oral y escrita.

La discusión de los talleres fue productiva, pues el 90 por ciento de los docentes alegaron que no dominaban estos contenidos con la actualización que poseen desde la didáctica del habla, y que son fundamentales en el trabajo con los estudiantes de la educación superior. Resumieron que todo lo que se instrumenta debe redundar en beneficio de la calidad de la instrucción y al mismo tiempo en la apropiación de conocimientos.

Estas razones permiten arribar a las consideraciones finales del estudio realizado.

\section{CONCLUSIONES}

La formación continua del profesional que trabaja en las universidades, requiere de una sistemática capacitación o superación que dé cuenta de la actualización de contenidos y su contextualización a través de las Ciencias de la Educación con énfasis la Pedagogía, la Didáctica, y la Psicología. Pero el docente debe asumir una autopreparación que permita a mediano y largo plazos nuevos modos de actuación en sus contextos educativos, para el logro de la calidad de los egresados.

Los talleres realizados como parte del trabajo metodológico y la superación, permiten profundizar en temáticas valiosas y necesarias para brindar atención de excelencia a los estudiantes de la educación superior, a partir de las debilidades que afectan la asimilación de los conocimientos, y al mismo tiempo, corroborar los niveles de desempeño de los docentes. 


\section{REFERENCIAS BIBLIOGRÁFICAS}

Álvarez de Zayas, C. M. (1999). La necesidad de la formación permanente en el profesorado". En nuestra escuela No 104. Madrid.

(1995). La Pedagogía Universitaria. Una experiencia cubana. La Habana: Pedagogía'95.

Antón A., P. (2010). Necesidades de formación y motivación del profesorado universitario para la aplicación de las propuestas metodológicas derivadas de la utilización de las Tecnologías de la Información y de la Comunicación e n la docencia. Madrid, España: Universidad Complutense de Madrid.

Correa D., R. (2009). Plan Nacional para el Buen Vivir 2013 - 2017 (2da. ed.) Quito: Secretaría Nacional de Planificación y Desarrollo - SENPLADES.

Chávez R., J., Suárez L., A., \& Permuy G., L. (2005). Acercamiento necesario a la Pedagogía General. La Habana, Cuba: Pueblo y Educación.

Day, C. (2005). Formar docentes: Cómo, cuándo y en qué condiciones aprende el profesorado. Obtenido de http://www.ciudaddelaeducacion.es/

Escudero, J. (1998). La Formación Permanente del Profesorado. Revista de Educación. Septiembre-Diciembre ( Vol. 317). Madrid, España.

Flores O., R. (s.f.). Hacia una pedagogía del conocimiento. (U. d. Antioquía,) Antioquía, Colombia.

Fuentes A., A., Borja M., et al. (2013). Propuesta de Capacitación. Curacao: Caribean International University.

Fuentes G., H. (s.f.). Dinámica del proceso docente educativo. (U. Oriente, Ed.) Santiago de Cuba: Cuba: Centro de estudios de Educación Superior "Manuel F. Gran".

Gastón, Pérez \& Otros. (1996). Metodología de la investigación educacional. Cuba. Editorial Pueblo y Educación.

Gorodokin, I. (s.f.). La formación docente y su relación con la epistemología. (E. I. d. Pringles, Editor). http://www.rieoei.org/deloslectores/1164Gorodokin.pdf

Horruitiner S., P. (2009). La Universidad Cubana modelo de Formación Curso: Estrategias de Aprendizaje en la Nueva Universidad Cubana. La Habana: Editorial Universitaria del Ministerio de Educación Superior. 
Medrano Rodríguez, H., \& Molina Granados, S. (2010). Desempeño profesional de docentes del Siglo XXI. (C. I. 2021, Ed.) Buenos Aires, Argentina: Instituto de Investigación, Innovación y Estudios de Posgrado para la Educación [IIIEPE].

Ortega, S. (2011). Formación Continua. (UNESCO-OREALC/CEPPE, Ed.) Proyecto Estratégico Regional sobre Docentes.

Presidencia de la República del Ecuador. (2010). Ley Orgánica de Educación Superior (LOES). Quito, Ecuador:

Pérez M. J., Lie concepción, A., \& Torres Retorta, D. (2020). Revista electrónica Odiseo de Pedagogía: Obtenido de La Pedagogía como Ciencia de la Educación: http://odiseo.com.mx/correos - lector/pedagogía-como-cienciaeducación.

Quezada, L., Grundmann, G., Expósito Verdejo, M., \& Valdez, L. (2001). Preparación y ejecución de talleres de capacitación: una guía práctica. Santo Domingo, Centro Cultural Poveda. República Dominicana. http://bibliotecavirtual.clacso.org.ar/Republica_Dominicana/ccp/201207310519 $\underline{03 / \mathrm{pr}}$

Sacristán, Gimeno. El currículo: un instrumento esencial para la formación de estudiantes universitarios. En revista Docencia e Investigación. Vol (20). España.

UNICEF. (2012). Formación Docente inicial y continua trabajando por la calidad de una educación con identidad propia. (E.-E. I. Bolivia-Ecuador-Perú, Ed.) Quito, Ecuador: Unai Sacona.

Varela Sierra, René. (2018). El proceso de formación del profesional en la educación superior basado en competencias: el desafío de su calidad en busca de una mayor integralidad de los egresados. Revista Roca \# 4, Vol (2 ). Bayamo. Cuba 
Ciencia Latina Revista Científica Multidisciplinar, Ciudad de México, México.

ISN 2707-2207 / ISSN 2707-2215 (en línea), noviembre-diciembre, 2021, Volumen 5, Número 6. https://doi.org/10.37811/cl_rcm.v5i6.1421 p14622 